\title{
Characterizations of continuous probability distributions occurring in physics and allied sciences by truncated moment
}

\author{
M. Ahsanullah ${ }^{1}$, M. Shakil ${ }^{2 *}$ \\ ${ }^{1}$ Rider University, Lawrenceville, NJ 08648, USA \\ ${ }^{2}$ Miami Dade College, Hialeah, FL 33012, USA \\ *Corresponding authorE-mail: mshakil@mdc.edu
}

Copyright () 2015 M. Ahsanullah, M. Shakil. This is an open access article distributed under the Creative Commons Attribution License, which permits unrestricted use, distribution, and reproduction in any medium, provided the original work is properly cited.

\begin{abstract}
A probability distribution can be characterized through various methods. Before a particular probability distribution model is applied to fit the real-world data, it is necessary to confirm whether the given continuous probability distribution satisfies the underlying requirements by its characterization. In this paper, characterizations of some continuous probability distributions occurring in physics and allied sciences have been established. We have considered the normal, Laplace, Lorentz, logistic, Boltzmann, Rayleigh, log-normal, Maxwell, Fermi-Dirac, and Bose-Einstein distributions, and characterized them by applying a truncated moment method; that is, by taking a product of reverse hazard rate and another function of the truncated point. It is hoped that the proposed characterizations will be useful for researchers in various fields of physics and allied sciences.
\end{abstract}

Keywords: Characterization; Continuous Probability Distribution; Reverse Hazard Rate; Truncated Moment.

\section{Introduction}

Many random phenomena in physics and allied sciences can be modelled through different continuous probability distributions, among them, normal, Laplace, Lorentz, Boltzmann, logistic, Rayleigh, log-normal, Maxwell, FermiDirac, and Bose-Einstein distributions are notable. For details on some of these distributions, the interested readers are referred to Johnson et al. [10], [11], Evans et al. [4], Balakrishnan and Nevzorov [3], and Ahsanullah, et al. [2], among others. As pointed out by Oldham, et al. [14], normal, logistic, and Lorentz (also known as the Cauchy) distributions have applications in chemical separation process, where the chromatographic peaks follow a normal distribution, and the electrochemical peaks follow a logistic distribution. The applications of normal distribution can also be found in statistical mechanics to describe the statistical behavior of a particle. On the other hand, the applications of the Lorentz and Boltzmann distributions can be found in nuclear magnetic resonance spectroscopy. Further, as pointed out by Oldham, et al. [14], the Boltzmann distribution describes the falloff in atmospheric pressure with height, as well as many other phenomena in which a force is opposed by thermal agitation. The speeds of gas molecules in two and threedimensional kinetic theory of gases follow the Rayleigh and Maxwell distributions respectively. The log-normal distribution is used to model the size distribution of granular materials. The Laplace distribution has applications in signal processing modeling. The statistical behaviors of subatomic particles, such as fermions (particles with halfinteger spin) and bosons (integer spin particles), can be described by the Fermi-Dirac and Bose-Einstein distributions respectively, see, for example, Kittel [12], Pathria and Beale [15], and Shepherd [17], among others.

Characterization of a probability distribution plays an important role in probability and statistics. Before a particular probability distribution model is applied to fit the real-world data, it is necessary to confirm whether the given continuous probability distribution satisfies the underlying requirements by its characterization. A probability distribution can be characterized through various methods, see, for example, Ahsanullah et al. [2], among others. In recent years, there has been a great interest in the characterizations of probability distributions by truncated moments. 
For example, the development of the general theory of the characterizations of probability distributions by truncated moment began with the work of Galambos and Kotz [5]. Further development on the characterizations of probability distributions by truncated moments continued with the contributions of many authors and researchers, among them Kotz and Shanbhag [13], Glänzel [6], [7], and Glänzel et al. [8], are notable. However, most of these characterizations are based on a simple relationship between two different moments truncated from the left at the same point. As pointed out by Glänzel [6], these characterizations may also serve as a basis for parameter estimation. In this paper, motivated by the importance of the normal, Laplace, Lorentz, logistic, Boltzmann, Rayleigh, log-normal, Maxwell, Fermi-Dirac, and Bose-Einstein distributions in physics and allied sciences, we have characterized these distributions by applying a truncated moment method; that is, by taking a product of reverse hazard rate and another function of the truncated point. It appears from the literature that our proposed characterizations would be the first attempt to characterize these commonly used continuous probability distributions occurring in physics and allied sciences in one place. The organization of the paper is as follows. In Section 2, we will state the assumptions and establish a lemma which will be needed for the characterizations by truncated moment method of these distributions. Section 3 contains our main results for the characterizations of the above commonly used continuous probability distributions of physics and allied sciences by truncated moment. The concluding remarks are presented in Section 4. In order to characterize the said distributions by applying a truncated moment method, we will use some special functions that are available in Abramowitz and Stegun [1], Gradshteyn and Ryzhik [9], Oldham et al. [14], and Prudnikov et al. [16], among others.

\section{A Lemma}

In this section, we will establish a lemma (Lemma 2.1) which will be useful in proving our main results for the proposed characterizations of the continuous probability distributions occurring in physics and allied sciences by truncated moment.

Lemma 2.1: Let $X$ be an absolutely continuous (with respect to Lebesgue measure) random variable with cumulative distribution function (cdf) $F(x)$ and the probability density function $(p d f) f(x)$. We assume $\alpha=\inf \{x \mid F(x)>0\}$, $\beta=\sup \{x \mid F(x)<1\}$, and $E(X)$ exists for all $x \in(\alpha, \beta)$. Then $E(X \mid X \leq x)=g(x) \eta(x)$, where $\eta(x)=\frac{f(x)}{F(x)}, g(x)$ is a differentiable function of $x$, and $\int_{\alpha}^{x} \frac{u-g^{\prime}(u)}{g(u)} d u$ is finite for all $x \in(\alpha, \beta)$, if $f(x)=c e^{\int_{\alpha}^{x} \frac{u-g^{\prime}(u)}{g(u)} d u}$, where c is determined by the condition $\int_{\alpha}^{\beta} f(x) d x=1$.

Proof of Lemma 2.1: Since $\frac{\int_{\alpha}^{x} u f(u) d u}{F(x)}=\frac{g(x) f(x)}{F(x)}$, we have

$\int_{\alpha}^{x} u f(u) d u=g(x) f(x)$. Differentiating both sides of the equation, we obtain

$x f(x)=g^{\prime}(x) f(x)+g(x) f^{\prime}(x)$.

On simplification, we get

$\frac{f^{\prime}(x)}{f(x)}=\frac{x-g^{\prime}(x)}{g(x)}$

Integrating the above equation, we obtain

$f(x)=c e^{\int_{\alpha}^{x} \frac{u-g^{\prime}(u)}{g(u)} d u}$

where $c$ is determined such that $\int_{\alpha}^{\beta} f(x) d x=1$.

This completes the proof of Lemma 2.1.

Remark 2.1: In this paper, we have presented the characterization of distributions by using the condition $E(X \mid X \leq x)$. The characterization of distributions by the condition $E(X \mid X \geq x)$ can similarly be done by using the following Lemma 2.2. 
Lemma 2.2: Let $X$ be an absolutely continuous (with respect to Lebesgue measure) random variable with cumulative distribution function (cdf) $F(x)$ and the probability density function (pdf) $f(x)$. We assume that $E(X)$ exists for all $x \in(\alpha, \beta)$. If $E(X \mid X \geq x)=g(x) r(x)$, where $r(x)=\frac{f(x)}{1-F(x)}$ and $g(x)$ is a differentiable function of $x$ for all $x \in(\alpha, \beta)$, then $f(x)=c e^{-\int \frac{x+g^{\prime}(x)}{g(x)} d x}$, provided $\int_{\alpha}^{x} \frac{u+g^{\prime}(u)}{g(u)} d u$ is finite for all $x \in(\alpha, \beta)$, and $c$ is determined by the condition $\int_{\alpha}^{\beta} f(x) d x=1$.

Proof of Lemma 2.2: The proof is similar to that of lemma 2.1.

\section{Characterizations of continuous probability distributions occurring in physics and allied sciences by truncated moment}

This section discusses our proposed characterizations by truncated moment for the normal, Laplace, Lorentz, Boltzmann, logistic, Rayleigh, log-normal, Maxwell, Fermi-Dirac, and Bose-Einstein distributions occurring in physics and allied sciences.

\subsection{Normal distribution}

For simplicity, without loss of generality, we will consider standard normal, $N(0,1)$, distribution: $f(x)=\frac{1}{\sqrt{2 \pi}} e^{-\frac{x^{2}}{2}}$, $-\infty<x<\infty$, of which characterization is provided in Theorem 3.1 below.

Theorem 3.1: Suppose that an absolutely continuous (with respect to Lebesgue measure) random variable $X$ has the cumulative distribution function (cdf) $F(x)$ and probability distribution function (pdf) $f(x)$ for $-\infty<x<\infty$ We assume that $f^{\prime}(x)$ and $E(X \mid X \leq x)$ exist for all $x,-\infty<x<\infty$. Then

$E(X \mid X \leq x)=g(x) \eta(x)$,

where

$\eta(x)=\frac{f(x)}{F(x)}$, and $g(x)=-1$,

if and only if

$f(x)=\frac{1}{\sqrt{2 \pi}} e^{-\frac{1}{2} x^{2}}, \quad \forall x \in(-\infty, \infty)$,

which is the probability density function of the standard normal distribution.

Proof: Suppose $f(x)=\frac{1}{\sqrt{2 \pi}} e^{-\frac{1}{2} x^{2}}, \quad \forall x \in(-\infty, \infty)$.

Then

$g(x)=\frac{\int_{-\infty}^{x} t \frac{1}{\sqrt{2 \pi}} e^{-\frac{t^{2}}{2}} d t}{\frac{1}{\sqrt{2 \pi}} e^{-\frac{x^{2}}{2}}}=-1$.

Consequently, $E(X \mid X \leq x)=g(x) \eta(x)$, where $\eta(x)=\frac{f(x)}{F(x)}$, and, hence the proof of "if" part of the Theorem 3.1 follows from Lemma 2.1. We will now prove the "only if" condition of the Theorem 3.1. Suppose that $g(x)=-1$.

Then, by Lemma 2.1, we easily have 
$\frac{f^{\prime}(x)}{f(x)}=\frac{x-g^{\prime}(x)}{g(x)}=-x$.

On integrating the above equation with respect to $x$, we easily have

$f(x)=c e^{-\frac{x^{2}}{2}}$,

where $c$ is a constant to be determined. Using the boundary condition $\int_{-\infty}^{\infty} f(x) d x=1$, we easily obtain $c=\frac{1}{\sqrt{2 \pi}}$.

Thus $f(x)=\frac{1}{\sqrt{2 \pi}} e^{-\frac{x^{2}}{2}},-\infty<x<\infty$, which is the probability density function of the standard normal distribution. This completes the proof of Theorem 3.1 .

\subsection{Laplace distribution}

For simplicity, without loss of generality, we will consider standard Laplace distribution: $f(x)=\frac{1}{2} e^{-|x|},-\infty<x<\infty$, of which characterization is provided in Theorem 3.2 below.

Theorem 3.2: Suppose that an absolutely continuous (with respect to Lebesgue measure) random variable $X$ has the cumulative distribution function (cdf) $F(x)$ and probability distribution function ( $p d f) f(x)$ for $-\infty<x<\infty$ We assume that $f^{\prime}(x)$ and $E(X \mid X \leq x)$ exist for all $x,-\infty<x<\infty$. Then

$E(X \mid X \leq x)=g(x) \eta(x)$,

where

$\eta(x)=\frac{f(x)}{F(x)}$,

and

$g(x)=x-1, x<0$,

$$
=-(1+x), x \geq 0,
$$

if and only if

$f(x)=\frac{1}{2} e^{-|x|}, \quad \forall x \in(-\infty, \infty)$,

which is the probability density function of the standard Laplace distribution.

Proof: Suppose $f(x)=\frac{1}{2} e^{-|x|}, \quad \forall x \in(-\infty, \infty)$.

Then, after integration and simplification, we obtain the following

$$
\begin{aligned}
g(x) & =\frac{\int_{-\infty}^{x} t f(t) d t}{f_{X}(x)} . \\
& =\left\{\begin{array}{l}
x-1, x<0, \\
-(1+x), x \geq 0 .
\end{array}\right.
\end{aligned}
$$

Consequently, $E(X \mid X \leq x)=g(x) \eta(x)$, where $\eta(x)=\frac{f(x)}{F(x)}$, and, hence the proof of "if" part of the Theorem 3.2 follows from Lemma 2.1.

We will now prove the "only if" condition of the Theorem 3.2. Suppose that 
$g(x)=\left\{\begin{array}{l}x-1, x<0, \\ -(1+x), x \geq 0 .\end{array}\right.$

Then, by Lemma 2.1, we easily have

$\frac{f^{\prime}(x)}{f(x)}=\frac{x-g^{\prime}(x)}{g(x)}=\left\{\begin{array}{l}1, x<0, \\ -1, x \geq 0,\end{array}\right.$

which, on integrating with respect to $x$, easily gives

$f(x)=c e^{-|x|}, \quad \forall x \in(-\infty, \infty)$,

where $c$ is a constant to be determined. Now, using the boundary condition $\int_{-\infty}^{\infty} f(x) d x=1$, we easily obtain $c=\frac{1}{2}$. Thus $f(x)=\frac{1}{2} e^{-|x|}, \quad-\infty<x<\infty$,

which is the probability density function of the standard Laplace distribution. This completes the proof of Theorem 3.2 .

\subsection{Lorentz (or Cauchy) distribution}

Since Lorentz (or Cauchy) distribution does not have finite moments of any order, we will consider, for simplicity, the truncated Cauchy distribution: $f(x)=\frac{1}{\tan ^{-1} a} \frac{1}{1+x^{2}},-a \leq x \leq a, 0<a<\infty$, of which characterization is provided in Theorem 3.3 below.

Theorem 3.3: Suppose that an absolutely continuous (with respect to Lebesgue measure) random variable $X$ has the cumulative distribution function ( $c d f) F(x)$ and probability distribution function ( $p d f$ ) $f(x)$ for $-a \leq x \leq a, 0<a<\infty$. We assume that $f^{\prime}(x)$ and $E(X \mid X \leq x)$ exist for all $x,-a \leq x \leq a, 0<a<\infty$. Then

$E(X \mid X \leq x)=g(x) \eta(x)$,

where

$\eta(x)=\frac{f(x)}{F(x)}$, and $g(x)=\frac{\frac{1}{2} \ln \left(x^{2}+1\right)}{\frac{1}{1+x^{2}}}$,

if and only if

$f(x)=\frac{1}{2 \tan ^{-1} a} \frac{1}{1+x^{2}},-a \leq x \leq a, 0<a<\infty$,

which is the probability density function of the truncated Cauchy distribution.

Proof: Suppose $f(x)=\frac{1}{2 \tan ^{-1} a} \frac{1}{1+x^{2}},-a \leq x \leq a, 0<a<\infty$. Then, after integration and simplification, we obtain the

following:

$$
\begin{aligned}
g(x) & =\frac{\int_{0}^{x} t f(t) d t}{f_{X}(x)} \\
& =\frac{\frac{1}{2} \ln \left(x^{2}+1\right)}{\frac{1}{1+x^{2}}} .
\end{aligned}
$$

Consequently, $E(X \mid X \leq x)=g(x) \eta(x)$, where $\eta(x)=\frac{f(x)}{F(x)}$, and, hence the proof of "if" part of the Theorem 3.3 follows from Lemma 2.1 .

We will now prove the "only if" condition of the Theorem 3.3. Suppose that 
$g(x)=\frac{\frac{1}{2} \ln \left(x^{2}+1\right)}{\frac{1}{1+x^{2}}}$.

Then, by Lemma 2.1, we easily have

$\frac{f^{\prime}(x)}{f(x)}=\frac{x-g^{\prime}(x)}{g(x)}=-\frac{2 x}{1+x^{2}}$,

from which, on integrating with respect to $x$, we get

$f(x)=c \frac{1}{1+x^{2}}, \quad-a \leq x \leq a, 0<a<\infty$,

where $c$ is a constant to be determined.

Now, using the boundary condition $\int_{-\infty}^{\infty} f(x) d x=1$, we easily obtain $c=\frac{1}{2 \tan ^{-1} a}$. Thus

$f(x)=\frac{1}{2 \tan ^{-1} a} \frac{1}{1+x^{2}}, \quad-a \leq x \leq a, 0<a<\infty$,

which is the probability density function of the truncated Cauchy distribution. This completes the proof of Theorem 3.3 .

\subsection{Boltzmann distribution}

For simplicity, without loss of generality, we will consider the Boltzmann distribution in the following simple form: $f(x)=\frac{1}{\sigma} e^{-\frac{x}{\sigma}}, \sigma>0, x \geq 0$, of which characterization is provided in Theorem 3.4 below. The Boltzmann distribution was formulated by the Austrian theoretical physicist, Ludwig Boltzmann (1844 - 1906) during his studies of the statistical mechanics of gases in thermal equilibrium, which was later investigated in its present form in 1902 by the American mathematical physicist, Josiah Willard Gibbs (1839 - 1903).

Theorem 3.4: Suppose that an absolutely continuous (with respect to Lebesgue measure) random variable $X$ has the cumulative distribution function $(c d f) F(x)$ and probability distribution function $(p d f) f(x)$ for $x \geq 0$. We assume that $f^{\prime}(x)$ and $E(X \mid X \leq x)$ exist for all $x, x \geq 0$. Then

$E(X \mid X \leq x)=g(x) \eta(x)$,

where

$\eta(x)=\frac{f(x)}{F(x)}$, and $g(x)=\sigma^{2} e^{\frac{x}{\sigma}} \gamma(2, \sigma x)$,

if and only if

$f(x)=\frac{1}{\sigma} e^{-\frac{x}{\sigma}}, \sigma>0, x \geq 0$,

which is the probability density function of the Boltzmann distribution.

Proof: Suppose $f(x)=\frac{1}{\sigma} e^{-\frac{x}{\sigma}}, \sigma>0, x \geq 0$. Then, after integration and using the definition of the incomplete gamma function, $\gamma(n, z)=\int_{0}^{z} u^{n-1} e^{-u} d u$, we easily obtain, after simplification, the following

$g(x)=\frac{\int_{0}^{x} t f(t) d t}{f_{X}(x)}=\sigma^{2} e^{\frac{x}{\sigma}} \gamma\left(2, \frac{x}{\sigma}\right)$.

Consequently, $E(X \mid X \leq x)=g(x) \eta(x)$, where $\eta(x)=\frac{f(x)}{F(x)}$, and, hence the proof of "if" part of the Theorem 3.4 follows from Lemma 2.1.

We will now prove the "only if" condition of the Theorem 3.4. Suppose that 
$g(x)=\sigma^{2} e^{\frac{x}{\sigma}} \gamma\left(2, \frac{x}{\sigma}\right)$.

Then, by Lemma 2.1, and noting that $\frac{\partial \gamma(\alpha, t)}{\partial t}=t^{\alpha-1} e^{-t}$, we easily have

$\frac{f^{\prime}(x)}{f(x)}=\frac{x-g^{\prime}(x)}{g(x)}=\frac{-1}{\sigma}$

On integrating the above equation with respect to $x$, we easily have

$f(x)=c e^{-\frac{x}{\sigma}}$,

where $c$ is a constant to be determined. Using the boundary condition $\int_{0}^{\infty} f(x) d x=1$, we easily obtain $c=\frac{1}{\sigma}$. Thus $f(x)=\frac{1}{\sigma} e^{-\frac{x}{\sigma}}, \sigma>0, x \geq 0$, which is the probability density function of the Boltzmann distribution. This completes the proof of Theorem 3.4.

\subsection{Rayleigh distribution}

In this sub-section, in Theorem 3.5, we will characterize the Rayleigh distribution which is given by $f(x)=\frac{\pi x}{2 \mu^{2}} e^{-\frac{\pi x^{2}}{4 \mu^{2}}}$, $\mu>0, x \geq 0$. The Rayleigh distribution was formulated by the British physicist, Baron John William Strutt (1842 1919), also known as Lord Rayleigh.

Theorem 3.5: Suppose that an absolutely continuous (with respect to Lebesgue measure) random variable $X$ has the cumulative distribution function $(c d f) F(x)$ and probability distribution function $(p d f) f(x)$ for $x \geq 0$. We assume that $f^{\prime}(x)$ and $E(X \mid X \leq x)$ exist for all $x, x \geq 0$. Then

$E(X \mid X \leq x)=g(x) \eta(x)$,

where

$\eta(x)=\frac{f(x)}{F(x)}$, and $g(x)=\frac{2 \mu^{2}\left[\mu \operatorname{erf}\left(\sqrt{\frac{\pi}{4 \mu^{2}}} x\right)-x e^{-\frac{\pi x^{2}}{4 \mu^{2}}}\right]}{\pi x e^{-\frac{\pi x^{2}}{4 \mu^{2}}}}$

if and only if

$f(x)=\frac{\pi x}{2 \mu^{2}} e^{-\frac{\pi x^{2}}{4 \mu^{2}}}, \mu>0, x \geq 0$

which is the probability density function of the Rayleigh distribution.

Proof: Suppose $f(x)=\frac{\pi x}{2 \mu^{2}} e^{-\frac{-\pi x^{2}}{4 \mu^{2}}}, \mu>0, x \geq 0$. Then, after integration and using the definition of the error function, $\operatorname{erf}(z)=\frac{2}{\sqrt{\pi}} \int_{0}^{2} e^{-t^{2}} d t$, we easily obtain, after simplification, the following:

$g(x)=\frac{\int_{0}^{x} t f(t) d t}{f_{X}(x)}=\frac{2 \mu^{2}\left[\mu \operatorname{erf}\left(\sqrt{\frac{\pi}{4 \mu^{2}}} x\right)-x e^{-\frac{\pi x^{2}}{4 \mu^{2}}}\right]}{\pi x e^{-\frac{\pi x^{2}}{4 \mu^{2}}}}$.

Consequently, $E(X \mid X \leq x)=g(x) \eta(x)$, where $\eta(x)=\frac{f(x)}{F(x)}$, and, hence the proof of "if" part of the Theorem 3.5 follows from Lemma 2.1 .

We will now prove the "only if" condition of the Theorem 3.5. Suppose that 
$g(x)=\frac{2 \mu^{2}\left[\mu \operatorname{erf}\left(\sqrt{\frac{\pi}{4 \mu^{2}} x}\right)-x e^{-\frac{\pi x^{2}}{4 \mu^{2}}}\right]}{\pi x e^{-\frac{\pi x^{2}}{4 \mu^{2}}}}$.

Then, by Lemma 2.1, and noting that $\frac{d}{d z} \operatorname{erf}(z)=\frac{2}{\sqrt{\pi}} e^{-z^{2}}$, we easily have, after simplification, the following:

$\frac{f^{\prime}(x)}{f(x)}=\frac{x-g^{\prime}(x)}{g(x)}=\frac{1}{x}-\frac{\pi x}{2 \mu^{2}}$.

On integrating the above equation with respect to $x$, we easily obtain, on simplification, the following:

$f(x)=c x e^{-\frac{\pi x^{2}}{4 \mu^{2}}}$,

where $c$ is a constant to be determined. Using the boundary condition $\int_{0}^{\infty} f(x) d x=1$, we easily obtain $c=\frac{\pi}{2 \mu^{2}}$.

Thus $f(x)=\frac{\pi x}{2 \mu^{2}} e^{-\frac{\pi x^{2}}{4 \mu^{2}}}, \mu>0, x \geq 0$, which is the probability density function of the Rayleigh distribution. This completes the proof of Theorem 3.5.

\subsection{Lognormal distribution}

In this sub-section, in Theorem 3.6, we will characterize the lognormal distribution, which is given by $f(x)=\frac{1}{x \sigma \sqrt{2 \pi}} e^{-\frac{1}{2}\left(\frac{h x-\mu}{\sigma}\right)^{2}},-\infty<\mu<\infty, \sigma>0, x>0$. For simplicity, we assume $\mu=0$ and $\sigma=1$. Then, we have $f(x)=\frac{1}{x \sqrt{2 \pi}} e^{-\frac{1}{2}(\ln x)^{2}}$, which we call as the standard lognormal distribution.

Theorem 3.6: Suppose that an absolutely continuous (with respect to Lebesgue measure) random variable $X$ has the cumulative distribution function (cdf) $F(x)$ and probability distribution function (pdf) $f(x)$ for $x>0$. We assume that $f^{\prime}(x)$ and $E(X \mid X \leq x)$ exist for all $x, x>0$. Then

$E(X \mid X \leq x)=g(x) \eta(x)$

where

$\eta(x)=\frac{f(x)}{F(x)}$, and $g(x)=\frac{\sqrt{\frac{\sqrt{2}}{2}} \frac{1}{2}\left(\operatorname{erf}\left(\sqrt{2}\left(\frac{1}{2} \ln x-\frac{1}{2}\right)\right)+11\right) x}{e^{-\frac{1}{2}(\ln x)^{2}}}$,

if and only if

$f(x)=\frac{1}{x \sqrt{2 \pi}} e^{-\frac{1}{2}(\ln x)^{2}}$,

which is the probability density function of the standard lognormal distribution.

Proof: Suppose $f(x)=\frac{1}{x \sqrt{2 \pi}} e^{-\frac{1}{2}(\ln x)^{2}}, x>0$. Then, after integration and using the definition of the error function, $\operatorname{erf}(z)=\frac{2}{\sqrt{\pi}} \int_{0}^{e^{-t^{2}} d t}$, we easily obtain, after simplification, the following:

$g(x)=\frac{\int_{0}^{x} t f(t) d t}{f_{X}(x)}=\frac{\sqrt{\frac{\sqrt{2}}{2}} \frac{1}{2}\left(\operatorname{erf}\left(\sqrt{2}\left(\frac{1}{2} \ln x-\frac{1}{2}\right)\right)+1\right) x}{e^{-\frac{1}{2} \ln x x^{2}}}$. 
Consequently, $E(X \mid X \leq x)=g(x) \eta(x)$, where $\eta(x)=\frac{f(x)}{F(x)}$, and, hence the proof of "if" part of the Theorem 3.6 follows from Lemma 2.1 .

We will now prove the "only if" condition of the Theorem 3.6. Suppose that

$g(x)=\frac{\int_{0}^{x} t f(t) d t}{f_{X}(x)}=\frac{\sqrt{\frac{\pi}{2}} e^{\frac{1}{2}}\left(\operatorname{erf}\left(\sqrt{2}\left(\frac{1}{2} \ln x-\frac{1}{2}\right)\right)+1\right) x}{e^{-\frac{1}{2}(\ln x)^{2}}}$.

Then, differentiating $g(x)$ with respect to $x$, using Lemma 2.1, and noting that $\frac{d}{d z} \operatorname{erf}(z)=\frac{2}{\sqrt{\pi}} e^{-z^{2}}$, we easily have, after simplification, the following:

$\frac{f^{\prime}(x)}{f(x)}=\frac{x-g^{\prime}(x)}{g(x)}=-\frac{1}{x}(1+\ln x)$.

On integrating the above equation with respect to $x$, we easily obtain, on simplification, the following:

$f(x)=c e^{-\ln x-\frac{1}{2}(\ln x)^{2}}=\frac{c}{x} e^{-\frac{1}{2}(\ln x)^{2}}$,

where $c$ is a constant to be determined. Using the boundary condition $\int_{0}^{\infty} f(x) d x=1$, we easily obtain $c=\frac{1}{\sqrt{2 \pi}}$.

Thus $f(x)=\frac{1}{x \sqrt{2 \pi}} e^{-\frac{1}{2}(\ln x)^{2}}, x>0$ which is the probability density function of the standard lognormal distribution. This completes the proof of Theorem 3.6.

\subsection{Maxwell distribution}

In this sub-section, in Theorem 3.7, we will characterize the Maxwell distribution, which is given by $f(x)=\frac{32 x^{2}}{\pi^{2} \mu^{3}} e^{-\frac{4 x^{2}}{\pi \mu^{2}}}$, $\mu>0, x \geq 0$.

Theorem 3.7: Suppose that an absolutely continuous (with respect to Lebesgue measure) random variable $X$ has the cumulative distribution function $(c d f) F(x)$ and probability distribution function ( $p d f) f(x)$ for $x \geq 0$. We assume that $f^{\prime}(x)$ and $E(X \mid X \leq x)$ exist for all $x, x \geq 0$. Then

$E(X \mid X \leq x)=g(x) \eta(x)$,

where

$\eta(x)=\frac{f(x)}{F(x)}$, and $g(x)=\frac{\frac{1}{\frac{1}{2}\left(\frac{\mu \sqrt{\sqrt{n}}}{2}\right)^{4} \gamma\left(2, \frac{4 x^{2}}{\pi \mu^{2}}\right)}}{x^{2} e^{-\frac{4 x^{2}}{\pi u^{2}}}}$,

if and only if

$f(x)=\frac{32 x^{2}}{\pi^{2} \mu^{3}} e^{-\frac{4 x^{2}}{\pi \mu^{2}}}$

which is the probability density function of the Maxwell distribution.

Proof: Suppose $f(x)=\frac{32 x^{2}}{\pi^{2} \mu^{3}} e^{-\frac{4 x^{2}}{\pi \mu^{2}}}, \mu>0, x \geq 0$. Then, after integration and using the definition of the incomplete gamma function, $\gamma(n, z)=\int_{0}^{z} u^{n-1} e^{-u} d u$, we easily obtain, after simplification, the following: 
$g(x)=\frac{\int_{0}^{x} t f(t) d t}{f_{X}(x)}=\frac{\frac{1}{2}\left(\frac{\mu \sqrt{5}}{2}\right)^{4} \gamma\left(2, \frac{4 x^{2}}{\pi \mu^{2}}\right)}{x^{2} e^{-\frac{4 x^{2}}{\pi \mu^{2}}}}$

Consequently, $E(X \mid X \leq x)=g(x) \eta(x)$, where $\eta(x)=\frac{f(x)}{F(x)}$, and, hence the proof of "if" part of the Theorem 3.7 follows from Lemma 2.1.

We will now prove the "only if" condition of the Theorem 3.7. Suppose that

$g(x)=\frac{\int_{0}^{x} t f(t) d t}{f_{X}(x)}=\frac{\frac{1}{\left(\frac{\mu(\sqrt{\pi})}{2}\right)^{4} \gamma\left(2, \frac{4 x^{2}}{u^{2}}\right)}}{x^{2} e^{-\frac{4 x^{2}}{\pi \mu^{2}}}}$.

Then, differentiating $g(x)$ with respect to $x$, using Lemma 2.1, and noting that $\frac{\partial \gamma(\alpha, t)}{\partial t}=t^{\alpha-1} e^{-t}$, we easily have, after simplification, the following:

$\frac{f^{\prime}(x)}{f(x)}=\frac{x-g^{\prime}(x)}{g(x)}=\frac{2}{x}-\frac{8 x}{\pi \mu^{2}}$.

On integrating the above equation with respect to $x$, we easily obtain, on simplification, the following

$f(x)=c e^{\ln x^{2}-\frac{4 x^{2}}{\pi \mu^{2}}}=c x^{2} e^{-\frac{4 x^{2}}{\pi \mu^{2}}}$,

where $c$ is a constant to be determined. Using the boundary condition $\int_{0}^{\infty} f(x) d x=1$, we easily obtain $c=\frac{32}{\pi^{2} \mu^{3}}$.

Thus, $f(x)=\frac{32 x^{2}}{\pi^{2} \mu^{3}} e^{-\frac{4 x^{2}}{\pi \mu^{2}}}, \mu>0, x \geq 0$, which is the probability density function of the Maxwell distribution. This completes the proof of Theorem 3.7.

\subsection{Logistic distribution}

In this sub-section, in Theorem 3.8, we will characterize the logistic distribution which is given by $f(x)=\frac{1}{4 s} \sec ^{2}\left(\frac{x-\mu}{2 s}\right)=\frac{e^{-\frac{x-\mu}{s}}}{s\left(1+e^{-\frac{x-\mu}{s}}\right)^{2}},-\infty<\mu<\infty, s>0,-\infty<x<\infty$. Some researchers also use an alternative parameterization of the logistic distribution by taking $s=\frac{\sigma \sqrt{3}}{\pi}, \sigma>0$. For simplicity, we assume $\mu=0$ and $s=1$. Then, we have $f(x)=\frac{e^{-x}}{\left(1+e^{-x}\right)^{2}},-\infty<x<\infty$, which we call as the standard logistic distribution.

Theorem 3.8: Suppose that an absolutely continuous (with respect to Lebesgue measure) random variable $X$ has the cumulative distribution function (cdf) $F(x)$ and probability distribution function ( $p d f) f(x)$ for $-\infty<x<\infty$. We assume that $f^{\prime}(x)$ and $E(X \mid X \leq x)$ exist for all $x,-\infty<x<\infty$. Then

$E(X \mid X \leq x)=g(x) \eta(x)$,

where

$\eta(x)=\frac{f(x)}{F(x)}$, and $g(x)=\frac{\frac{x^{x}}{1+e^{x}}-\ln \left(1+e^{x}\right)}{\frac{e^{-x}}{\left(1+e^{-x}\right)^{2}}}$,

if and only if

$f(x)=\frac{e^{-x}}{\left(1+e^{-x}\right)^{2}},-\infty<x<\infty$, 
which is the probability density function of the standard logistic distribution.

Proof: Suppose $f(x)=\frac{e^{-x}}{\left(1+e^{-x}\right)^{2}},-\infty<x<\infty$. Then, after integration, we easily obtain, on simplification, the following

$g(x)=\frac{\int_{-\infty}^{x} t \frac{e^{-t}}{\left(1+e^{-t}\right)^{2}} d t}{\frac{e^{-x}}{\left(1+e^{-x}\right)^{2}}}=\frac{\frac{x e^{x}}{1+e^{x}}-\ln \left(1+e^{x}\right)}{\frac{e^{-x}}{\left(1+e^{-x}\right)^{2}}}$.

Consequently, $E(X \mid X \leq x)=g(x) \eta(x)$, where $\eta(x)=\frac{f(x)}{F(x)}$, and, hence the proof of "if" part of the Theorem 3.8 follows from Lemma 2.1.

We will now prove the "only if" condition of the Theorem 3.8. Suppose that

$g(x)=\frac{\int_{-\infty}^{x} t \frac{e^{-t}}{\left(1+e^{-t}\right)^{2}} d t}{\frac{e^{-x}}{\left(1+e^{-x}\right)^{2}}}=\frac{\frac{x e^{x}}{1+e^{x}}-\ln \left(1+e^{x}\right)}{\frac{e^{-x}}{\left(1+e^{-x}\right)^{2}}}$.

Then, differentiating $g(x)$ with respect to $x$, and using Lemma 2.1, we easily have, on simplification, the following:

$g^{\prime}(x)=x-g(x) \frac{e^{-x}-1}{e^{-x}+1}$.

Consequently, we have

$\frac{f^{\prime}(x)}{f(x)}=\frac{x-g^{\prime}(x)}{g(x)}=\frac{e^{-x}-1}{e^{-x}+1}$

On integrating the above equation with respect to $x$, we easily obtain, on simplification, the following

$f(x)=c \frac{e^{-x}}{\left(1+e^{-x}\right)^{2}}$,

where $c$ is a constant to be determined. Using the boundary condition $\int_{-\infty}^{\infty} f(x) d x=1$, we easily obtain $c=1$. Thus, $f(x)=\frac{e^{-x}}{\left(1+e^{-x}\right)^{2}},-\infty<x<\infty$, which is the probability density function of the standard logistic distribution. This completes the proof of Theorem 3.8.

\subsection{Fermi-Dirac distribution}

In this sub-section, in Theorem 3.9, we will characterize the Fermi-Dirac distribution which is given by $f(x)=\frac{\alpha \beta}{\ln (1+\beta)\left(e^{\alpha x}+\beta\right)},-1<\beta<0$ or $\beta>0, \alpha>0, x \geq 0$.

Theorem 3.9: Suppose that an absolutely continuous (with respect to Lebesgue measure) random variable $X$ has the cumulative distribution function (cdf) $F(x)$ and probability distribution function ( $p d f) f(x)$ for $x \geq 0$. We assume that $f^{\prime}(x)$ and $E(X \mid X \leq x)$ exist for all $x, x \geq 0$. Then

$E(X \mid X \leq x)=g(x) \eta(x)$,

where

$\eta(x)=\frac{f(x)}{F(x)}$, and $g(x)=\frac{\frac{1}{2}\left(\sum_{j=0}^{\infty}(-1)^{j} \frac{x^{2}{ }_{1} F_{1}(2,3, j \alpha x)}{\beta^{j+1}}\right)}{\frac{1}{e^{\alpha x}+\beta}}$,

if and only if 
$f(x)=\frac{\alpha \beta}{\ln (1+\beta)\left(e^{\alpha x}+\beta\right)},-1<\beta<0$ or $\beta>0, \alpha>0, x \geq 0$,

which is the probability density function of the Fermi-Dirac distribution.

Proof: Suppose $f(x)=\frac{\alpha \beta}{\ln (1+\beta)\left(e^{\alpha x}+\beta\right)},-1<\beta<0$ or $\beta>0, \alpha>0, x \geq 0$. Then, using the binomial expansion, $(1+w)^{-s}=\sum_{k=0}^{\infty} \frac{(s)_{k}(-w)^{k}}{k !}$, for any real value of $s$, where $(s)_{k}=\frac{\Gamma(s+k)}{\Gamma(s)}=s(s+1) \ldots(s+k-1),(s \neq 0)$, and $(s)_{0}=1$, denote the Pochhammer symbol, and the integral $\int_{0}^{x} e^{v} e^{b t} d t=\frac{x^{v+1}}{v+1}{ }_{1} F_{1}(v+1, v+2, b x), v>-1$, see Oldham, et al. [14], Eq. 26:10:14, page 246, we easily obtain, after integration and on simplification, the following:

$g(x)=\frac{\int_{0}^{x} t \frac{\alpha \beta}{\ln (1+\beta)\left(e^{\alpha t}+\beta\right)} d t}{\frac{\alpha \beta}{\ln (1+\beta)\left(e^{\alpha x}+\beta\right)}}=\frac{\frac{1}{2}\left(\sum_{j=0}^{\infty}(-1)^{j} \frac{x^{2}{ }_{1} F_{1}(2,3, j \alpha x)}{\beta^{j+1}}\right)}{\frac{1}{e^{\alpha x}+\beta}}$,

where ${ }_{1} F_{1}(a, c ; z)=\sum_{j=0}^{\infty} \frac{(a)_{j}}{(c)_{j}} z^{j}$ is known as the Kummer or degenerate hypergeometric function; see, for example, Abramowitz and Stegun [1], Gradshteyn and Ryzhik [9], Oldham et al. [14], and Prudnikov et al. [16], among others.

Consequently, $E(X \mid X \leq x)=g(x) \eta(x)$, where $\eta(x)=\frac{f(x)}{F(x)}$, and, hence the proof of "if" part of the Theorem 3.9 follows from Lemma 2.1 .

We will now prove the "only if" condition of the Theorem 3.9. Suppose that

$g(x)=\frac{\int_{0}^{x} \frac{\alpha \beta}{\ln (1+\beta)\left(e^{\alpha t+\beta)}\right.} d t}{\frac{\alpha \beta}{\ln (1+\beta)\left(e^{\alpha x}+\beta\right)}}=\frac{\frac{1}{2}\left(\sum_{j=0}^{\infty}(-1)^{j} \frac{x^{2}{ }_{1} F_{1}(2,3, j \alpha x)}{\beta^{j+1}}\right)}{\frac{1}{e^{\alpha x}+\beta}}=\frac{P(x)}{Q(x)}$, say,

where

$Q(x)=\frac{1}{e^{\alpha x}+\beta}$,

and

$P(x)=\frac{1}{2}\left(\sum_{j=0}^{\infty}(-1)^{j} \frac{x^{2}{ }_{1} F_{1}(2,3, j \alpha x)}{\beta^{j+1}}\right)$

$=\int_{0}^{x} t Q(t) d t$

Now, differentiating $g(x)=\frac{P(x)}{Q(x)}$ with respect to $x$, we obtain, after simplification, the following:

$g^{\prime}(x)=x-g(x) \frac{Q^{\prime}(x)}{Q(x)}$,

that is,

$\frac{x-g^{\prime}(x)}{g(x)}=\frac{Q^{\prime}(x)}{Q(x)}$.

Thus, using Lemma 2.1 in the above equation, we have

$$
\frac{f^{\prime}(x)}{f(x)}=\frac{x-g^{\prime}(x)}{g(x)}=\frac{Q^{\prime}(x)}{Q(x)}=\frac{-\alpha e^{\alpha x}}{e^{\alpha x}+\beta} .
$$

On integrating the above equation with respect to $x$, we easily obtain, on simplification, the following: 
$f(x)=c e^{\ln \left(\frac{1}{e^{\alpha x}+\beta}\right)}=c \frac{1}{e^{\alpha x}+\beta}$,

where $c$ is a constant to be determined. Using the boundary condition $\int_{0}^{\infty} f(x) d x=1$, we easily obtain, on integration and simplification, the following:

$c=\frac{\alpha \beta}{\ln (1+\beta)}$.

Thus, $f(x)=\frac{\alpha \beta}{\ln (1+\beta)\left(e^{\alpha x}+\beta\right)},-1<\beta<0$ or $\beta>0, \alpha>0, x \geq 0$,

which is the probability density function of the Fermi-Dirac distribution. This completes the proof of Theorem 3.9.

\subsection{Bose-Einstein distribution}

In this sub-section, in Theorem 3.10, we will characterize the Bose-Einstein distribution, which is given by $f(x)=\frac{-\alpha \beta}{\ln (1-\beta)\left(e^{\alpha x}-\beta\right)}, 0<\beta<1, \alpha>0, x \geq 0$.

Theorem 3.10: Suppose that an absolutely continuous (with respect to Lebesgue measure) random variable $X$ has the cumulative distribution function $(c d f) F(x)$ and probability distribution function (pdf) $f(x)$ for $x \geq 0$ We assume that $f^{\prime}(x)$ and $E(X \mid X \leq x)$ exist for all $x, x \geq 0$. Then

$E(X \mid X \leq x)=g(x) \eta(x)$

where

$\eta(x)=\frac{f(x)}{F(x)}$, and $g(x)=\frac{\frac{1}{2}\left(\sum_{j=0}^{\infty} \frac{x^{2}{ }_{1} F_{1}(2,3, j \alpha x)}{\beta^{j+1}}\right)}{\frac{1}{e^{\alpha x}-\beta}}$,

if and only if

$f(x)=\frac{-\alpha \beta}{\ln (1-\beta)\left(e^{\alpha x}-\beta\right)}, 0<\beta<1, \alpha>0, x \geq 0$,

which is the probability density function of the Bose-Einstein distribution.

Proof: Suppose $f(x)=\frac{-\alpha \beta}{\ln (1-\beta)\left(e^{\alpha x}-\beta\right)}, 0<\beta<1, \alpha>0, x \geq 0$. Then, using the binomial expansion, $(1-w)^{-s}=\sum_{k=0}^{\infty} \frac{(s)_{k}(w)^{k}}{k !}$, for any real value of $s$, where $(s)_{k}=\frac{\Gamma(s+k)}{\Gamma(s)}=s(s+1) \ldots(s+k-1),(s \neq 0)$, and $(s)_{0}=1$, denote the Pochhammer symbol, and the integral $\int_{0}^{x} t^{v} e^{b t} d t=\frac{x^{v+1}}{v+1}{ }_{1} F_{1}(v+1, v+2, b x), v>-1$, see Oldham, et al. [14], Eq. 26:10:14, page 246, we easily obtain, after integration and on simplification, the following:

$g(x)=\frac{\int_{0}^{x} t \frac{-\alpha \beta}{\ln (1-\beta)\left(e^{\alpha x}-\beta\right)} d t}{\frac{-\alpha \beta}{\ln (1-\beta)\left(e^{\alpha x}-\beta\right)}}=\frac{\frac{1}{2}\left(\sum_{j=0}^{\infty} \frac{x^{2} F_{1}(2,3, j \alpha x)}{\beta^{j+1}}\right)}{\frac{1}{e^{\alpha x}-\beta}}$,

where ${ }_{1} F_{1}(a, c ; z)=\sum_{j=0}^{\infty} \frac{(a)_{j}}{(c)_{j}} z^{j}$ is known as the Kummer or degenerate hypergeometric function; see, for example, Abramowitz and Stegun [1], Gradshteyn and Ryzhik [9], Oldham et al. [14], and Prudnikov et al. [16], among others.

Consequently, $E(X \mid X \leq x)=g(x) \eta(x)$, where $\eta(x)=\frac{f(x)}{F(x)}$, and, hence the proof of "if" part of the Theorem 3.10 follows from Lemma 2.1.

We will now prove the "only if" condition of the Theorem 3.10. Suppose that 
$g(x)=\frac{\int_{0}^{x} t \frac{-\alpha \beta}{\ln (1-\beta)\left(e^{\alpha x}-\beta\right)} d t}{\frac{-\alpha \beta}{\ln (1-\beta)\left(e^{\alpha x}-\beta\right)}}=\frac{\frac{1}{2}\left(\sum_{j=0}^{\infty} \frac{x^{2}{ }_{1} F_{1}(2,3, j \alpha x)}{\beta^{j+1}}\right)}{\frac{1}{e^{\alpha x}-\beta}}=\frac{M(x)}{N(x)}$, say,

where

$N(x)=\frac{1}{e^{\alpha x}-\beta}$,

and

$M(x)=\frac{1}{2}\left(\sum_{j=0}^{\infty} \frac{x^{2}{ }_{1} F_{1}(2,3, j \alpha x)}{\beta^{j+1}}\right)$

$=\int_{0}^{x} t N(t) d t$

Now, differentiating $g(x)=\frac{M(x)}{N(x)}$ with respect to $x$, we obtain, after simplification, the following:

$g^{\prime}(x)=x-g(x) \frac{N^{\prime}(x)}{N(x)}$,

that is,

$\frac{x-g^{\prime}(x)}{g(x)}=\frac{N^{\prime}(x)}{N(x)}$.

Thus, using Lemma 2.1 in the above equation, we have

$$
\frac{f^{\prime}(x)}{f(x)}=\frac{x-g^{\prime}(x)}{g(x)}=\frac{N^{\prime}(x)}{N(x)}=\frac{-\alpha e^{\alpha x}}{e^{\alpha x}-\beta} .
$$

On integrating the above equation with respect to $x$, we easily obtain, on simplification, the following:

$f(x)=c e^{\ln \left(\frac{1}{e^{\alpha x}-\beta}\right)}=c \frac{1}{e^{\alpha x}-\beta}$,

where $c$ is a constant to be determined. Using the boundary condition $\int_{0}^{\infty} f(x) d x=1$, we easily obtain, on integration and simplification, the following:

$c=\frac{-\alpha \beta}{\ln (1-\beta)}$.

Thus, $f(x)=\frac{-\alpha \beta}{\ln (1-\beta)\left(e^{\alpha x}-\beta\right)}, 0<\beta<1, \alpha>0, x \geq 0$,

which is the probability density function of the Bose-Einstein distribution. This completes the proof of Theorem 3.10.

\section{Concluding remarks}

Characterization of a probability distribution plays an important role in probability and statistics. In this paper, we have considered some continuous probability distributions occurring in physics and allied sciences, namely, the normal, Laplace, Lorentz, logistic, Boltzmann, Rayleigh, log-normal, Maxwell, Fermi-Dirac, and Bose-Einstein distributions, and have presented their characterizations by using a truncated moment method; that is, by using the condition $E(X \mid X \leq x)$. The characterization of distributions by the condition $E(X \mid X \geq x)$ can similarly be done by using the Lemma 2.2. It is hoped that the proposed characterizations will be useful for researchers in various fields of physics and allied sciences. 


\section{Acknowledgment}

The authors would like to thank the editor for helpful suggestions and review process, which improved the quality and presentation of the paper.

\section{References}

[1] Abramowitz, M., and Stegun, I. A. (1970). Handbook of Mathematical Functions, with Formulas, Graphs and Mathematical Tables. Dover, New York.

[2] Ahsanullah, M., Kibria, B. M. G. and Shakil, M. (2014). Normal and Student's t Distributions and Their Applications. Atlantis Press, Paris, France. http://dx.doi.org/10.2991/978-94-6239-061-4.

[3] Balakrishnan, N., and Nevzorov, V. B. (2003). A Primer on Statistical distributions. John Wiley \& Sons, New Jersey. http://dx.doi.org/10.1002/0471722227.

[4] Evans, M. et al (2000). Statistical Distributions. John Wiley \& Sons, New York.

[5] Galambos, J., and Kotz, S. (1978). Characterizations of probability distributions. A unified approach with an emphasis on exponential and related models, Lecture Notes in Mathematics, 675, Springer, Berlin.

[6] Glänzel, W. (1987). A characterization theorem based on truncated moments and its application to some distribution families, Mathematical Statistics and Probability Theory (Bad Tatzmannsdorf, 1986), Vol. B, Reidel, Dordrecht, 75-84.

[7] Glänzel, W. (1990) some consequences of a characterization theorem based on truncated moments, Statistics, 21, 613-618 http://dx.doi.org/10.1080/02331889008802273.

[8] Glänzel, W., Telcs, A. and Schubert, A. (1984) Characterization by truncated moments and its application to Pearson-type distributions, Z. Wahrsch. Verw. Gebiete, 66, 173-183 http://dx.doi.org/10.1007/BF00531527.

[9] Gradshteyn, I. S. and Ryzhik, I. M. (1980). Table of Integrals, Series, and Products (6th Printing). Academic Press, San Diego.

[10] Johnson, N. L., Kotz, S. and Balakrishnan, N. (1994). Continuous Univariate Distributions, Vol. 1, Second Edition, John Wiley and Sons, New York, 1994.

[11] Johnson, N. L., Kotz, S. and Balakrishnan, N. (1995). Continuous Univariate Distributions: Vol. 2, Wiley, New York.

[12] Kittel, C. (2004). Elementary Statistical Physics. Dover Publications, New York, USA.

[13] Kotz, S. and Shanbhag, D.N. (1980). Some new approaches to probability distributions. Advances in Applied Probability, 12, $903-921$. http://dx.doi.org/10.2307/1426748.

[14] Oldham, K. B., Myland, J., and Spanier, J. (2009). An Atlas of Functions with Equator, the Atlas Function Calculator. Springer, New York, USA.

[15] Pathria, R. K., and Beale, P. D. (2011). Statistical Mechanics. Academic Press, New York, USA.

[16] Prudnikov, A. P., Brychkov, Y. A. and Marichev, O. I. (1986). Integrals and Series, Volumes 1, 2, and 3, Gordon and Breach Science Publishers. Amsterdam.

[17] Shepherd, P. J. (2013). A Course in Theoretical Physics. John Wiley \& Sons, Ltd., New York, USA. http://dx.doi.org/10.1002/9781118516911. 